Supporting Information

\title{
Assessment of Anisotropic Semiconductor Nanorod and Nanoplatelet Heterostructures with Polarized Emission for Liquid Crystal Display Technology
}

Patrick D. Cunningham, ${ }^{l}$ João B. Souza Jr., ${ }^{1,2}$ Igor Fedin, ${ }^{l}$ Chunxing She, ${ }^{1}$ Byeongdu Lee, ${ }^{3}$ and Dmitri V. Talapin ${ }^{1,4 *}$

${ }^{1}$ Department of Chemistry and James Franck Institute, University of Chicago, Chicago, Illinois, 60637, USA

${ }^{2}$ Instituto de Química de São Carlos, Universidade de São Paulo - USP, Colloidal Materials Group, CP 780, 13566-590, São Carlos - SP, Brazil

${ }^{3}$ Advanced Photon Source, Argonne National Laboratory, Argonne, IL 60439, USA

${ }^{4}$ Center for Nanoscale Materials, Argonne National Laboratory, Argonne, IL 60439, USA

E-mail: dvtalapin@uchicago.edu 


\section{CdSe and CdSe@CdS heterostructure synthesis and post-synthetic modifications}

High quantum yields were preserved through addition of excess ligand, such as n-octylamine, to improve colloidal stability of nanorod solutions upon storage. ${ }^{1}$ In order to retain high quantum yield over extended period of time, sample exposure to oxygen has to be minimized prior to shell growth by washing solutions of CdSe "seeds" in inert atmosphere. Through additional annealing and surface passivation with $\mathrm{Cd}$ and $\mathrm{S}$ precursors, quantum yields approaching $100 \%$ can be achieved for $\mathrm{CdSe} / \mathrm{CdS}$ spherical core-shells ${ }^{2}$ and dot-in-rod samples ${ }^{3}$ according to recent studies by the Bawendi group.

The importance of a good interface between $\mathrm{CdSe} @ \mathrm{CdS}$ was emphasized. With respect to washing in oxygen environment, the photoluminescence of CdSe dropped off significantly. It is for this reason that CdSe "seeds" were washed and stored in the glovebox before epitaxial growth. This washing likely prevents surface oxidation of Se and leads to good epitaxial growth on a surfaces with homogenous composition.

Storage of CdSe nanocrystals in excess amines has shown to drastically enhance photoluminescence. For this reason, our CdSe@CdS nanorods were stored in excess of octylamine. The octylamine will serve to passivate the surface of the CdS layer, eliminating surface traps which will inhibit radiative recombination. This ligand also likely preserves colloidal stability. In a representative set of particles, quantum yield increased from 51\% to $72 \%$ over 1 week of storage in the presence of octylamine. Thus, this post-synthetic modification increases the quality of our samples for incorporation into films. The narrowest spectral linewidths could be obtained through size fractionation.

$512 \mathrm{~nm}$ (4 ML) CdSe NPLs of three lateral dimensions. To synthesize $512 \mathrm{~nm}$ CdSe NPLs, we followed a recipe from with slight modifications. ${ }^{4}$ Namely, we degassed $170 \mathrm{mg}$ $\mathrm{Cd}(\text { myristate })_{2}$ in $15 \mathrm{ml} \mathrm{ODE}$ at the room temperature for $30 \mathrm{~min}$, then we added $12 \mathrm{mg}$ of Se powder and degassed the system for $30 \mathrm{~min}$ at $90^{\circ} \mathrm{C}$. Then we rapidly heated up the reaction mixture to $240^{\circ} \mathrm{C}$ under nitrogen. At $190^{\circ} \mathrm{C}$, when the solution turned orange-red, we introduced $40 \mathrm{mg}$ of finely ground $\mathrm{Cd}(\mathrm{OAc})_{2}$. We ran the reaction at $240^{\circ} \mathrm{C}$ for $5 \mathrm{~min}$, then quickly cooled down the reaction mixture to $150^{\circ} \mathrm{C}$ and allowed it to further cool down. At $70^{\circ} \mathrm{C}$, we injected a solution of $2 \mathrm{ml}$ of oleic acid in $10 \mathrm{ml}$ ODE. When the system cooled down to the room 
temperature, we precipitated the NPLs by centrifugation and re-dispersed them in hexane.

By taking aliquots, we noticed the NPLs acquired their spectroscopic features soon after the introduction of $\mathrm{Cd}(\mathrm{OAc})_{2}$. We hypothesize that the NPLs acquire their target thickness fast and then grow laterally. We can thus terminate the recipe prematurely to obtain shorter $512 \mathrm{~nm}$ NPLs.

550 nm (5 ML) CdSe NPLs. To synthesize $550 \mathrm{~nm}$ CdSe NPLs, we followed exactly the recipe from the source. ${ }^{4}$ Namely, we degassed $170 \mathrm{mg} \mathrm{Cd}(\text { myristate })_{2}$ in $14 \mathrm{ml} \mathrm{ODE}$ at the room temperature, heated up the reaction mixture under nitrogen, at $240^{\circ} \mathrm{C}$ injected $12 \mathrm{mg}$ of $\mathrm{Se}$ powder pre-dispersed in $1 \mathrm{ml}$ ODE by sonication, in $20 \mathrm{~s}$ introduced $60 \mathrm{mg}$ of finely ground $\mathrm{Cd}(\mathrm{OAc})_{2}$, ran the reaction for $10 \mathrm{~min}$, cooled down the reaction mixture, and at $80^{\circ} \mathrm{C}$ injected 2 $\mathrm{ml}$ of oleic acid in $15 \mathrm{ml}$ ODE. When the suspension reached the r.t., we centrifuged it without a non-solvent and re-dispersed the NPLs in hexane.

A remark. $512 \mathrm{~nm}$ and $550 \mathrm{~nm}$ CdSe NPLs tend to form stacks of 5 - 30 NPLs. A recent work by B. Guzelturk et al. ${ }^{5}$ demonstrates a 3-picosecond homo-FRET rate in stacks of $512 \mathrm{~nm} \mathrm{CdSe}$ NPLs. One defective NPL in a stack can serve as an exciton drain and lower thus the PL efficiency of the sample. We modified the recipes for the regular $512 \mathrm{~nm}$ and $550 \mathrm{~nm}$ NPLs so that, after the completion of the reaction at $240^{\circ} \mathrm{C}$, we collected the hot reaction mixture with a glass syringe and injected it into a solution of oleic acid in hexane. This modification lowered the degree of NPL stacking and increased the PL efficiency of NPL solutions.

\section{Characterization of anisotropic nanostructures in solutions}

Size fractionation ${ }^{5}$ can easily reduce spectral widths of dot-in-rod samples down to $25 \mathrm{~nm}$ or less. To compare these data with homogeneous linewidths for these samples, we carried out photoluminescence excitation (PLE) measurements on colloidal samples (Figures S1-S2).

Dot-in-rod samples with emission FWHM of $23 \mathrm{~nm}$ had the linewidth of the first excitonic peak of about $20 \mathrm{~nm}$ in PLE spectra measured with $1 \mathrm{~nm}$ slits (Figure S2a). Rod-in-rod samples with ensemble linewidths of $33 \mathrm{~nm}$ had homogenous linewidths of $25 \mathrm{~nm}$ (Figure S2b). These numbers provide estimates for potentially attainable PL FWHM and color purity of dot-in-rods and rod-in-rods ensembles. It is also possible that fractionation of the colloidal solutions of 
"seeds" could provide even further minimization of spectral linewidths. These data also show that PL FWHM of CdSe/CdS dot-in-rods and rod-in-rods are on par with spherical $\mathrm{CdSe} / \mathrm{CdS}$ QDs. ${ }^{2}$
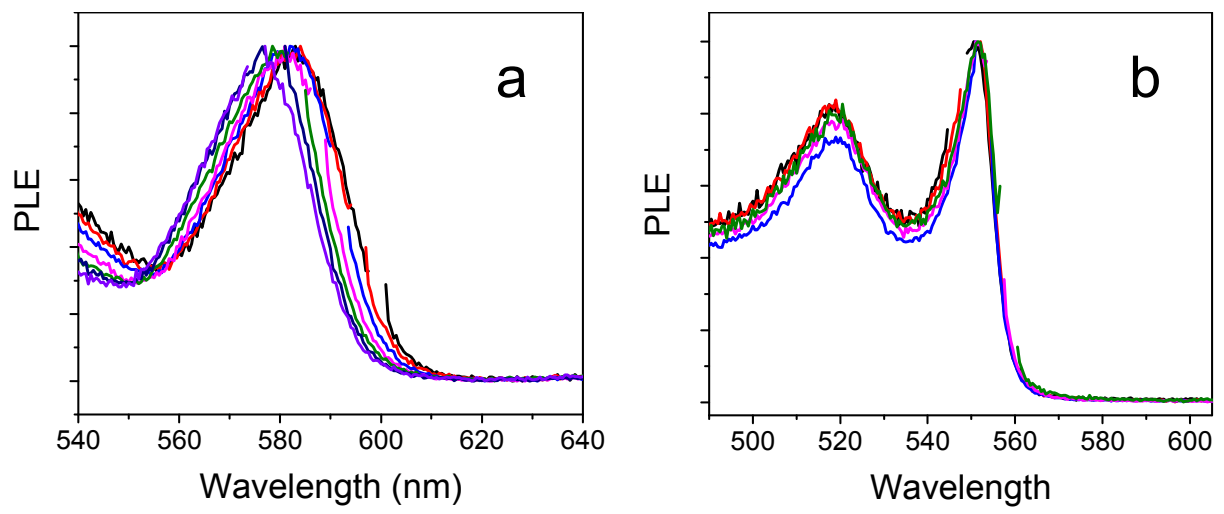

Figure S1. (a) PLE spectra of CdSe/CdS nanorods taken at different emission wavelengths (slit width $=1 \mathrm{~nm}$ ) reveal significant inhomogenous broadening coming from particle size distribution. (b) PLE spectra of CdSe nanoplatelets emitting at 550nm, taken at different emission wavelengths (slit width $=1 \mathrm{~nm}$ ) reveal negligible inhomogenous broadening in NPLs.
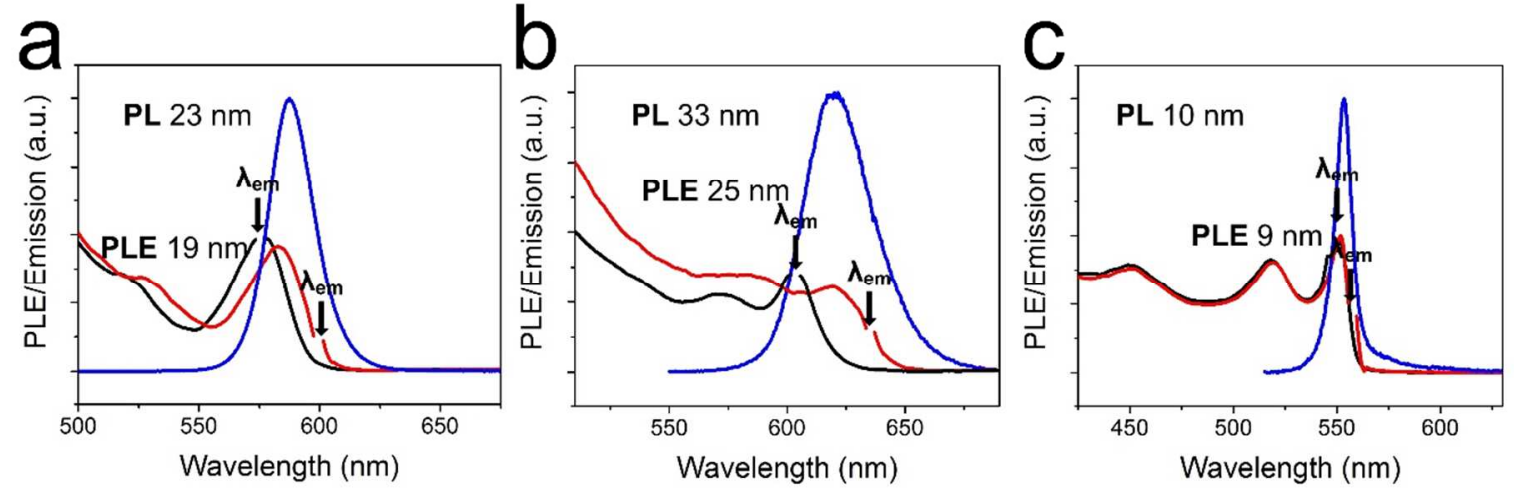

Figure S2. Linewidths estimated from PLE spectra measured at high (black curves) and low energy (red curves) wavelengths of the emission profiles (blue curves) for (a) $\mathrm{CdSe} / \mathrm{CdS}$ dot-inrods (orange emitting rods without size fractionation from Figure 2d), (b) $\mathrm{CdSe} / \mathrm{CdS}$ rod-in-rods $(5.9 \times 23 \mathrm{~nm}$ with $3.4 \times 8.6 \mathrm{~nm}$ core), (e) CdSe nanoplatelets emitting at $550 \mathrm{~nm}$. The excitation and emission slits were fixed at $1 \mathrm{~nm}$. 
Determination of PL quantum yields.

Most of the general procedure for determination of quantum yield is detailed in the Methods section. For the determination of PL quantum yield we used fresh Rhodamine 6G, Coumarin 153, and Oxazine 170 perchlorate dyes in ethanol solution. In general, the samples were excited at the wavelength where the absorption spectra of the organic dye and nanocrystal solution were equal (Figure S3). The optical density of the solution at this wavelength was kept between 0.03 and 0.05 .

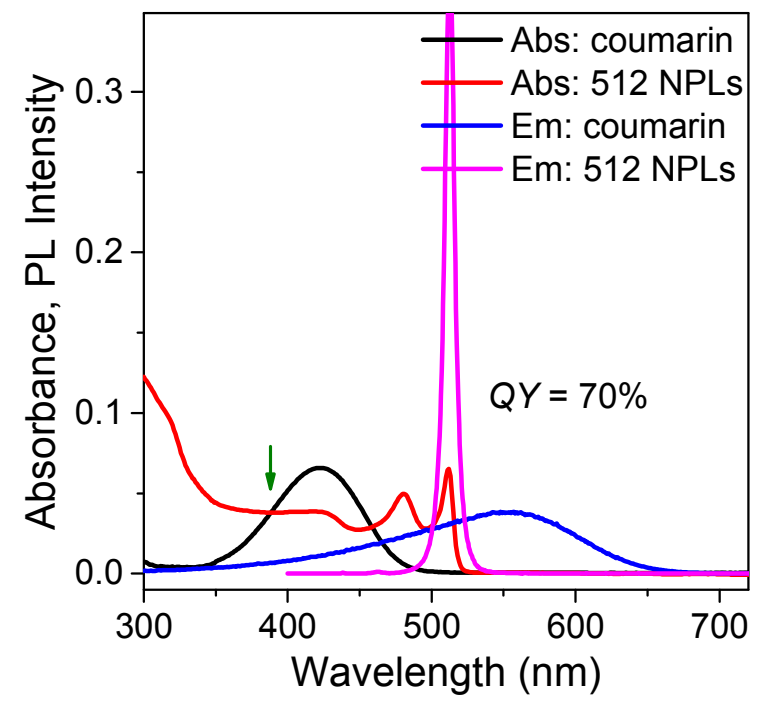

Figure S3. Determination of photoluminescence quantum yield in CdSe NPLs in comparison with an organic standard dye.
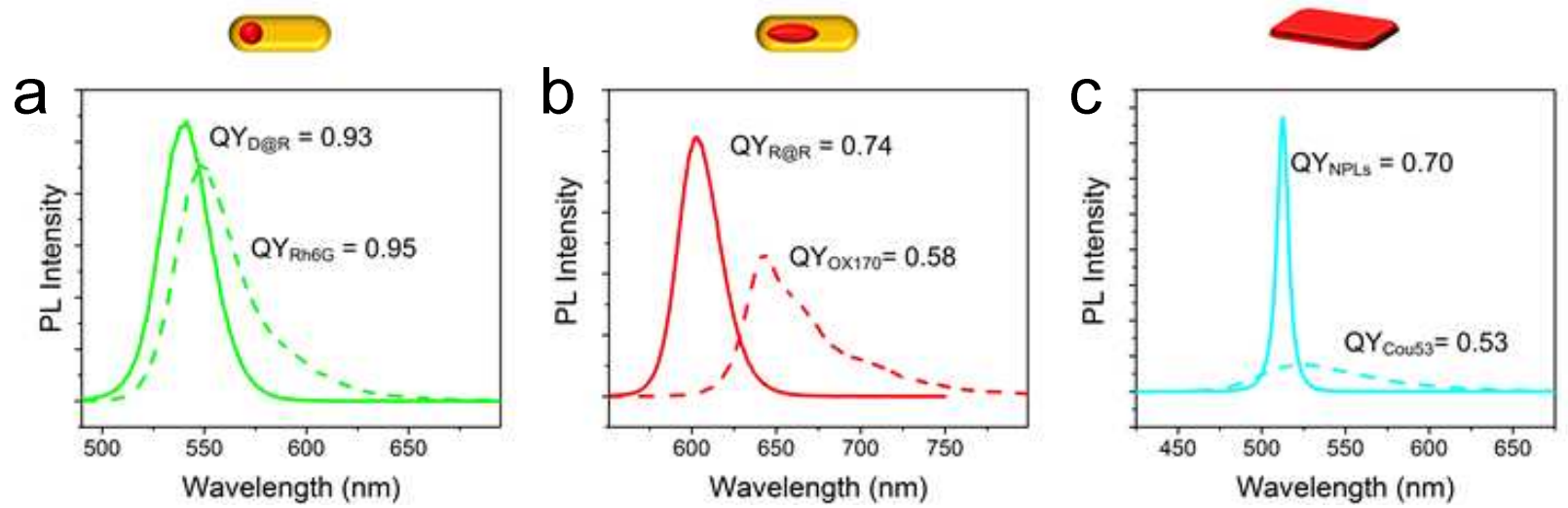
Figure S4. Determination of nanostructure photoluminescence quantum yields by comparison with organic standard dyes ${ }^{5}$ for (a) CdSe@CdS dot-in-rods, (b) CdSe@CdS rod-in-rods and (c) CdSe nanoplatelets. 


\section{Nanocrystal anisotropy in a colloidal solution}

The detailed procedures and theory related to optical anisotropy of solution samples are available in the main text and previous studies. ${ }^{6,7}$ Anisotropies were measured in a JobinYvonne Fluorolog 3 using computer controlled polarizers. In general, the optical density in the region of interest for the sample was kept below 0.05 and their absorption spectra were taken prior to measurement. The anisotropies were recorded at 1 or $2 \mathrm{~nm}$ increments over regions blueshifted from the band edge. As many samples have a much higher extinction coefficient at higher energies, the regions blueshifted in the spectra were not recorded. This was done to avoid the inner filter effect and saturating the detector signal. In case of CdSe nanoplatelets, measuring the "band-edge" anisotropy required particular caution due to a small Stokes shift that increases possibility of excitation scatter in the emission channel.

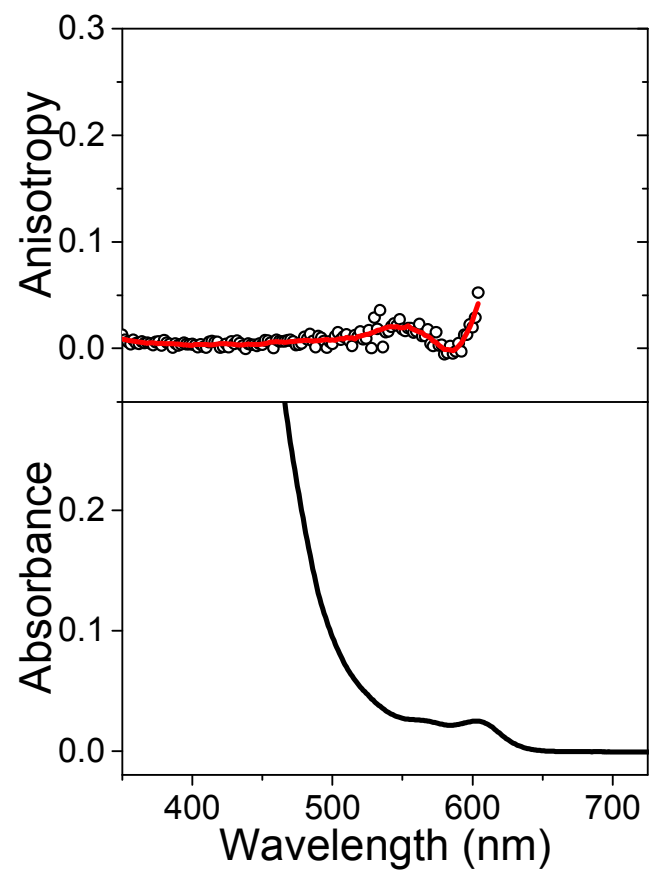

Figure S5. Anisotropy (open circles, red curve) and absorption (black curve) of nearly spherical $\mathrm{CdSe} / \mathrm{CdS}$ core-shell quantum dots.

Dot-in-rod samples with medium sized cores $(\sim 3-4 \mathrm{~nm})$ and very long and thin shells provided the largest values of anisotropy at both band edge and blue excitation energies (Figure 3f). 
Samples with large cores had higher band edge anisotropies than those with smaller cores but their higher energy anisotropies are similar-likely due to the similar size and aspect ratio of their CdS shells (Figure 3f).

In order to optimize anisotropies for both red and green emission colors, one should focus on the physical dimensions of core-shell nanorods with respect to their inherent optical anisotropies rather than their susceptibility to efficient alignment. For green nanorods, the emission polarization will be lower due to the smaller gap in the optically active excitonic states; ${ }^{8}$ therefore it is likely that a maximum degree of linear emission polarization of 0.5 can be obtained for dot-in-rod structures with smaller cores. The best way to optimize polarization within these structures is to grow sufficiently thin and long shells by increasing precursor concentrations in shell-growth synthesis. Additionally, we can make the CdSe cores slightly larger ( 2.5-3.0 $\mathrm{nm}$ in diameter) in order to obtain a suitable fine exciton structure for increased polarization. ${ }^{8}$ However, this polarization may compromise pure green emission, as the increase of core size would shift the peak emission to the wavelengths greater than $550 \mathrm{~nm}$. For redemitting nanorods, the effect of $\mathrm{CdS}$ shell on the emission wavelength is weaker. The polarization can be optimized by using a sufficiently large CdSe rod as the seed, which should increase the energy spacing between the optically active excitonic states. Ideally, rod-in-rod heterostructures should be used for red emission. This increased spacing enhances the polarization and the growth of a thin and long shell would not be as critical.
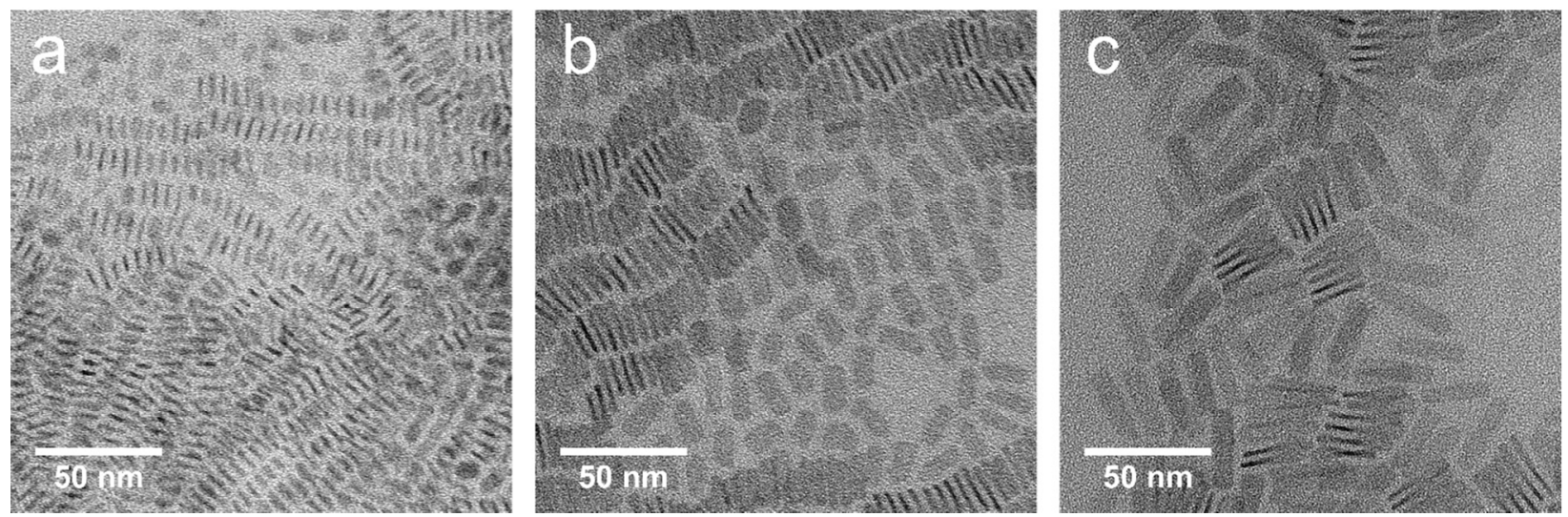

Figure S6. Transmission electron microscope images of CdSe NPLs with $1.2 \mathrm{~nm}$ thickness and different lateral dimensions: (a) "short" (b) "medium" (c) "long”. 


\section{Polymer Screening}

To maximize brightness and macroscopic alignment of $\mathrm{CdSe} / \mathrm{CdS}$ nanostructures, we sought a polymer medium which dispersed nanocrystals, had co-solubility in nonpolar organic solvents, and had a glass transition temperature $\left(\mathrm{T}_{\mathrm{g}}\right)$ above room temperature. Such a medium would allow us to obtain a composite with fixed alignment, suitable concentration, homogeneity and optical clarity. After screening a number of candidates (Table S1), ${ }^{5}$ poly(butyl-co-isobutyl methacrylate) (PBiBMA) was chosen for solubility in chloroform and toluene, combined with good viscoelasticity at relatively mild temperatures $\left(\mathrm{T}_{\mathrm{g}}=35^{\circ} \mathrm{C}\right)$. In the case of NPLs, we payed special attention to prevent aggregation and NPL stacking by using freshly prepared solutions.

Table S1. Preliminary results of polymers tested for dispersion and stretching of composites.

\begin{tabular}{|c|c|c|c|}
\hline Polymer & $\mathrm{T}_{\mathrm{g}}\left({ }^{\circ} \mathrm{C}\right)$ & Description & $\begin{array}{l}\text { Best Result } \\
\text { Polarized } \\
\text { Emission }\end{array}$ \\
\hline $\begin{array}{l}\text { poly (butyl methacrylate) } \\
\text { (PBMA) }\end{array}$ & 15 & $\begin{array}{l}\text { Strong plastic film, resistant to } \\
\text { stretching at RT; stretchable in } \\
50{ }^{\circ} \mathrm{C} \text { water bath }\end{array}$ & $1.7: 1$ \\
\hline $\begin{array}{l}\text { poly (butyl-co-isobutyl methacrylate) } \\
\text { (PBiBMA) }\end{array}$ & 35 & $\begin{array}{l}\text { Resistant to stretching at RT; } \\
\text { stretchable in } 60^{\circ} \mathrm{C} \text { water bath }\end{array}$ & $2.3: 1$ \\
\hline $\begin{array}{l}\text { poly (benzyl methacrylate) } \\
\text { (PBzMA) }\end{array}$ & 55 & $\begin{array}{l}\text { Brittle, difficult to delaminate } \\
\text { from glass substrate even at } \\
\text { elevated temperatures }\end{array}$ & N/A \\
\hline $\begin{array}{l}\text { poly (cyclohexyl methacrylate) } \\
\text { (PCyMA) }\end{array}$ & 83 & $\begin{array}{l}\text { Somewhat brittle, cracks easily } \\
\text { when stretched at RT and over } \\
\text { a heat gun }\end{array}$ & N/A \\
\hline $\begin{array}{l}\text { polystyrene-block-polybutadiene-block- } \\
\text { polystyrene } \\
\text { (PSBS) }\end{array}$ & N/A & $\begin{array}{l}\text { Spongy rubber film; stretchable } \\
\text { at room temperature; relaxes } \\
\text { rapidly }\end{array}$ & $1.4: 1$ \\
\hline
\end{tabular}

Additionally, several films were prepared using the same batch of CdSe@CdS nanorods to determine the effect of additives on dispersibility and alignment. Films additives were used as $1.5 \%$ wt. of n-octylamine (OctAm) and 1.5\% wt. of n-trioctylphosphine (TOP). Qualitatively, the 
films containing TOP maintained their bright color but appeared cloudy, whereas films with no additives and OctAm were optically clear. The SAXS patterns from the resulting films indicated that samples prepared with TOP had aggregates, whereas films with no additives had fewer and films with OctAm appeared to have almost none (Figure S7). After stretching, the nanorods w TOP are much more strongly aligned and have small orientation angles. The nanorods with no additives present are still well aligned, whereas those with OctAm show weaker alignment. It is possible that in the presence of octylamine the interaction of nanorods with the polymer brush is minimal. The presence of aggregates in the film with TOP could be responsible for an increase in alignment due to cooperative effects.

Non-stretched
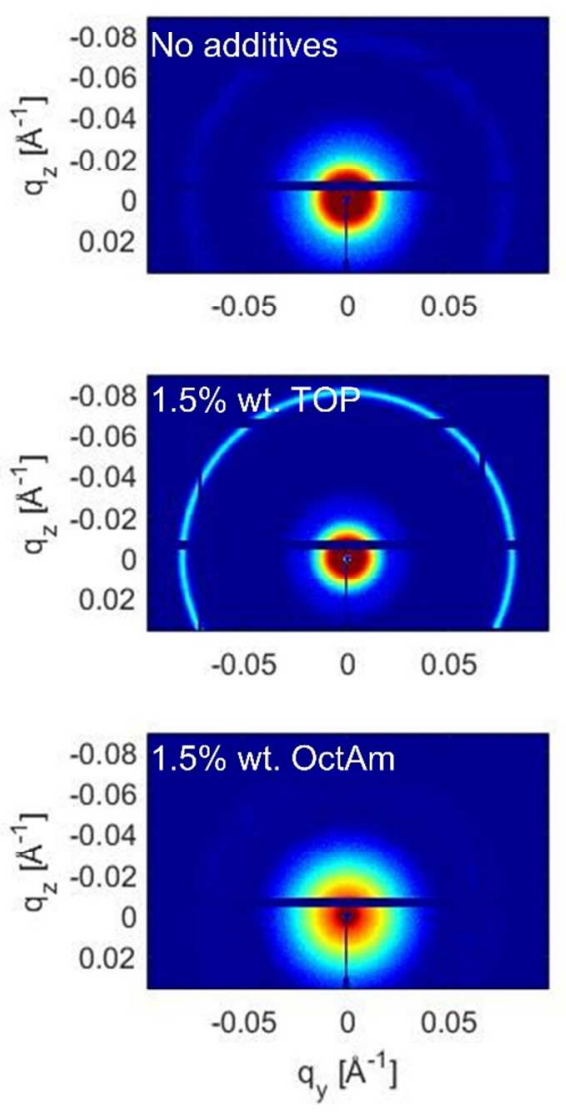

Stretched
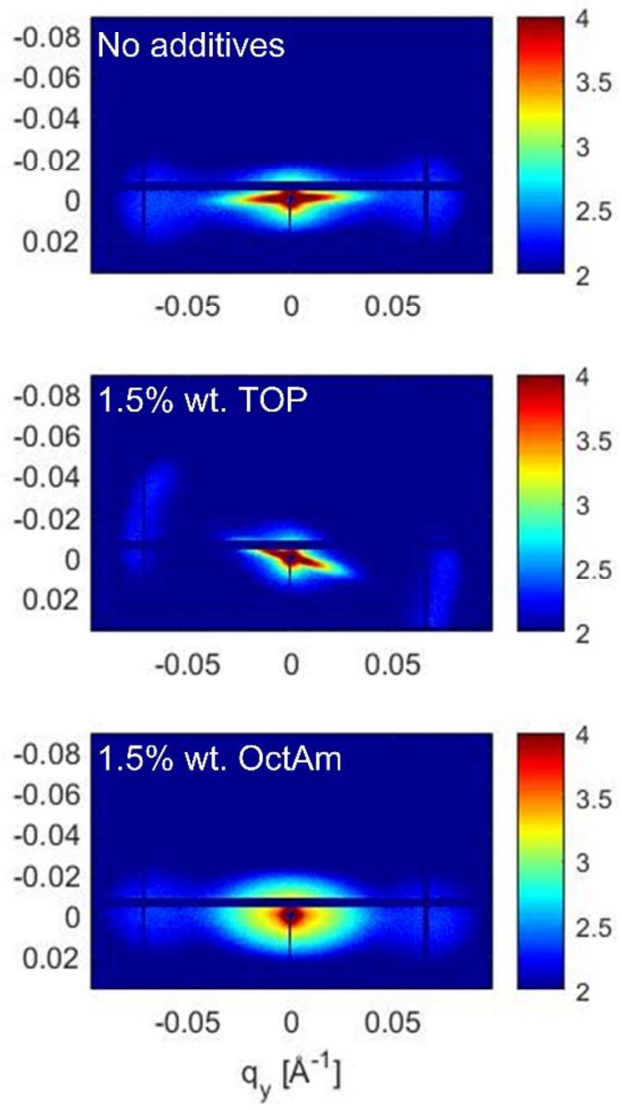

Figure S7. SAXS patterns of films before and after stretching with different additives. Clear differences in intensity and orientation are seen in each case. 


\section{Additional Optical Characterization}
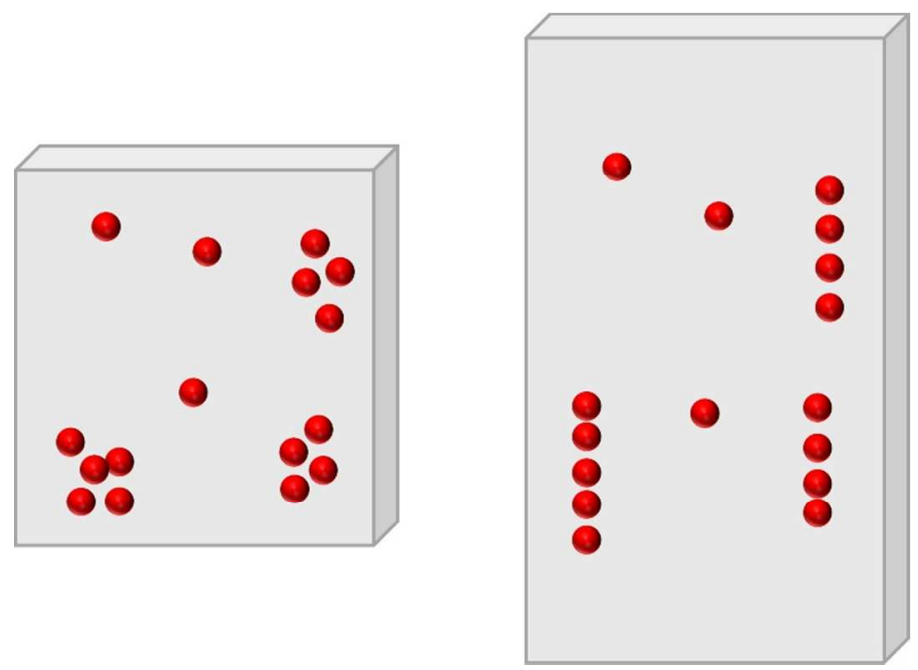

Figure S8. Representation of origin of dielectric screening of aggregates with respect to stretching of spherical quantum dot films.

Table S2. Summary of SAXS alignment data compared with emission polarization data

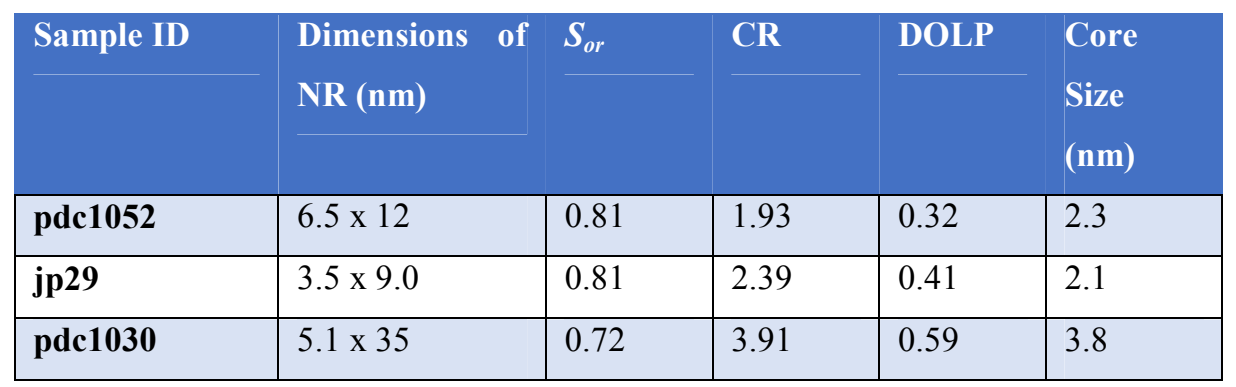

For measurement of absorption of polarized light we used a Glan-Taylor Polarizer to filter out light from the UV-Vis lamp in the Cary 5000. To assure that any polarization response we measured was not an artifact, we measured the polarization dependence of an isotropic emitting film sample. The error in optical density was 0.003 throughout the spectra, leading to a larger percent error at lower optical density regions (Figure S9). 


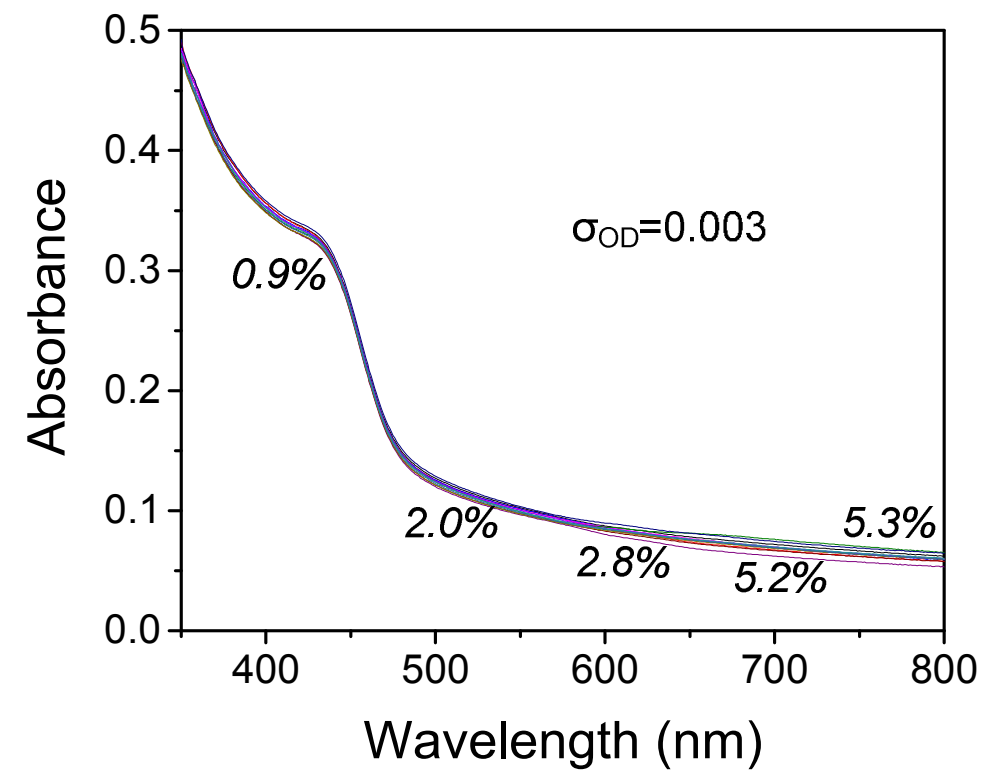

Figure S9. Error propagation in optical density for measurement of polarized absorbance at angles between 0-90.

A high concentration nanorod-PBiBMA composite was prepared using our typical procedure for dropcasting polymer films. The resulting film was stretched at elevated temperatures and photoselected emission spectra were recorded. The film was then placed between two glass slides and wrapped with foil and placed on a hotplate set to $90{ }^{\circ} \mathrm{C}$. The internal temperature is estimated to be $60{ }^{\circ} \mathrm{C}$. The film was left to age for 1 day and its emission spectra were recorded again. The film was then placed on a hotplate in the same fashion and left at an internal temperature of $100{ }^{\circ} \mathrm{C}$ for 5 days (hotplate $=130{ }^{\circ} \mathrm{C}$ ). The spectra were again recorded and the appearance was noted.

After one day at mild conditions, a slight change in visible appearance was observed - the color went from slightly opaque orange to a more clear yellow. The polarization of the sample was mostly maintained with the contrast ratio decreasing from 2.75 to 2.65 . After nearly a week at the harsher conditions, the film appeared to form domains where there were likely minor changes in thickness due to melting and reforming. The contrast ratio only drops to 2.22 in this case. At such harsh conditions, the alignment of nanorods is only slightly perturbed. 


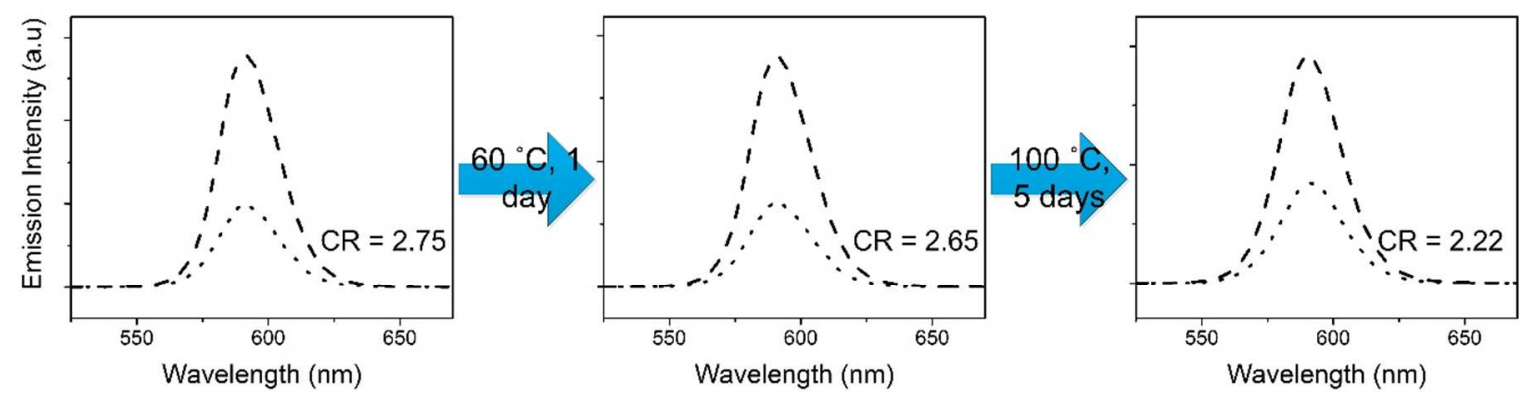

Figure S10. Emission spectra of stretched film of CdSe@CdS nanorods in polymer thermally aged over 6 days. Emission spectra were obtained with no photoselection (solid), polarized parallel to stretching axis (dashed), and polarized perpendicular to stretching axis (dotted).

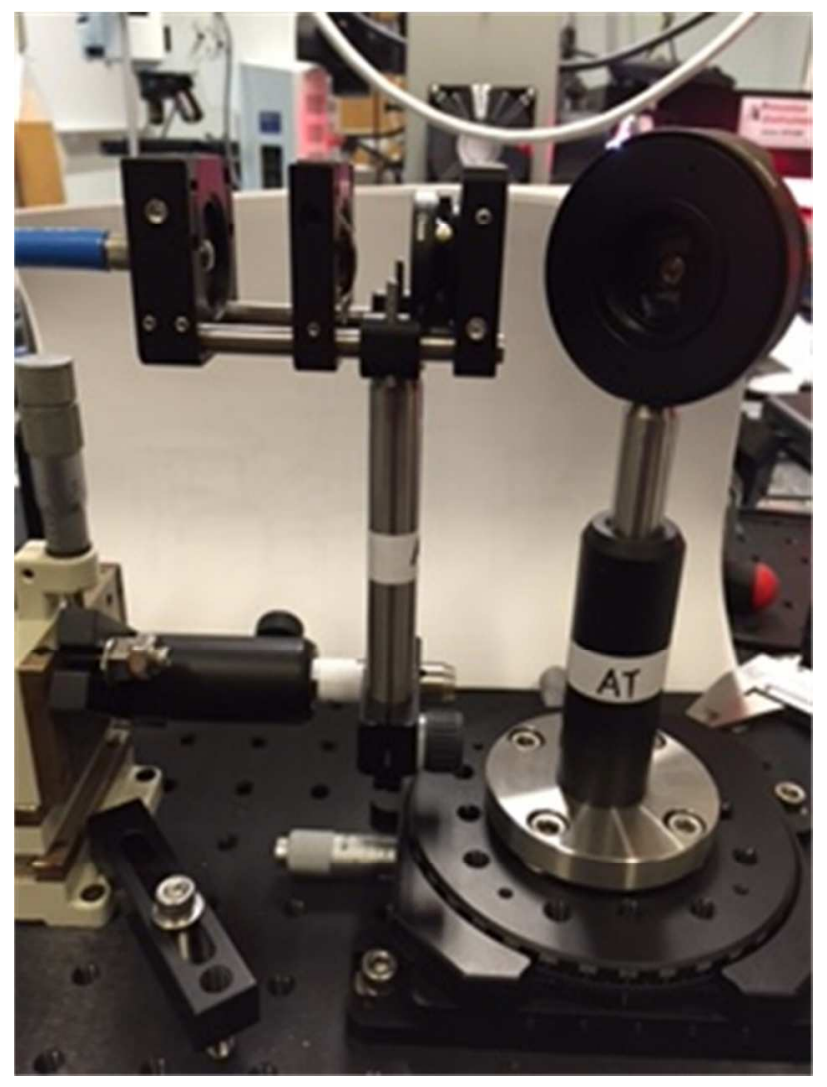

Figure S11. Optical setup for measuring directionality of emission in films. Film is excited from behind the sample and the holder has two rotational degrees of freedom at the base and the head. Emission is collected through focused collection optics. 


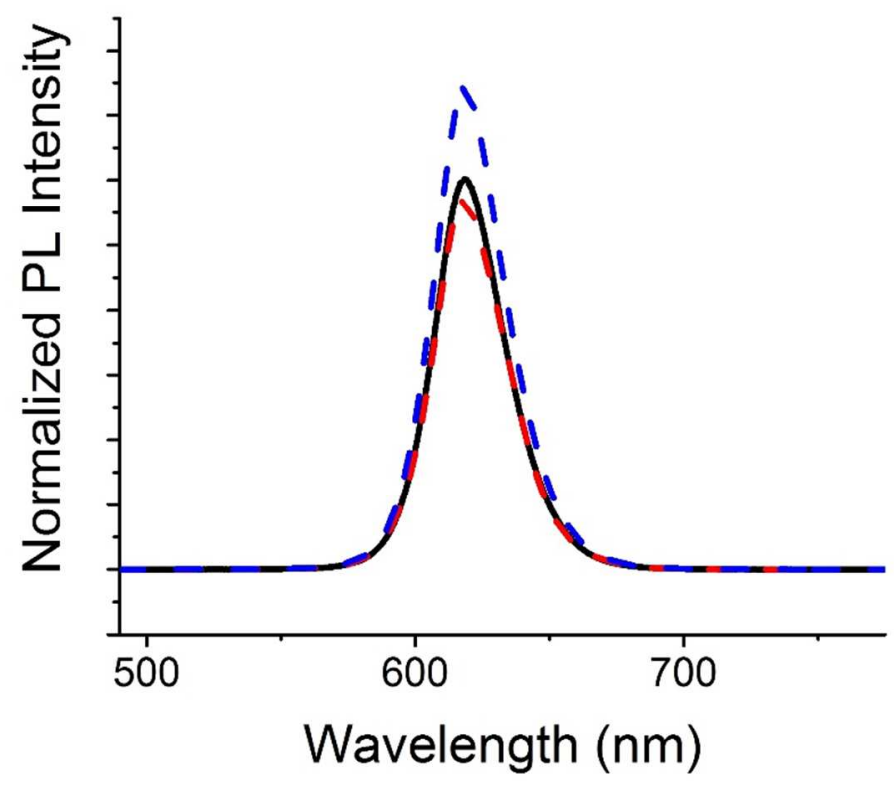

Figure S12. Film emission normalized to optical density with respect to non-polarized light of films before and after stretching for nearly spherical CdSe/CdS core-shell QDs. The optical anisotropy measured in solution for these QDs is shown in Figure S5.
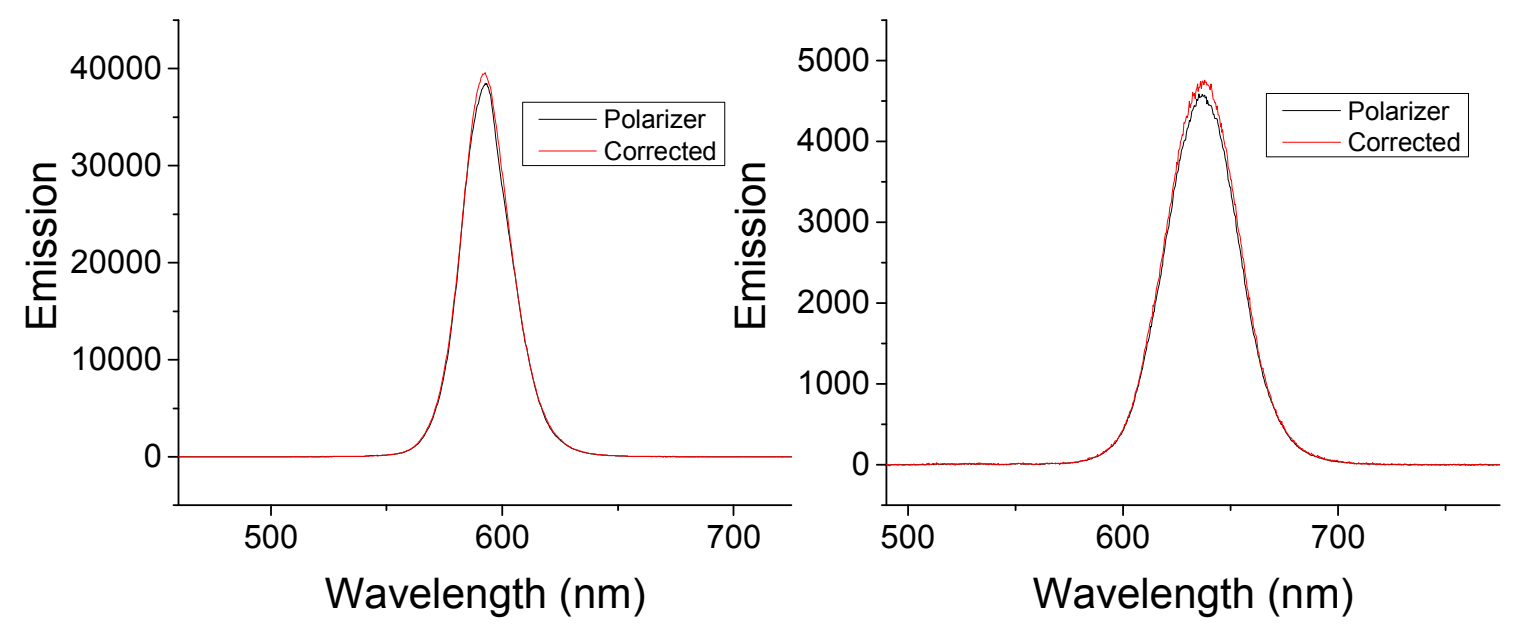

Figure S13. Comparison of isotropic emitting films containing disordered CdSe@CdS dot-inrod nanostructures. Polarizer filters were placed atop films and resulting spectra were compared to those without a polarizer filter but corrected with a factor representing the transmittance of the polarizer filter. 


\section{Supplementary References}

1. Bullen, C.; Mulvaney, P. The Effects of Chemisorption on the Luminescence of CdSe Quantum Dots. Langmuir 2006, 22, 3007-3013.

2. Chen, O.; Zhao, J.; Chauhan, V. P.; Cui, J.; Wong, C.; Harris, D. K.; Wei, H.; Han, H. S.; Fukumura, D.; Jain, R. K.; Bawendi, M. G. Compact High-Quality CdSe-CdS Core-Shell Nanocrystals with Narrow Emission Linewidths and Suppressed Blinking. Nat. Mater. 2013, 12 , 445-51.

3. Coropceanu, I.; Rossinelli, A.; Caram, J. R.; Freyria, F. S.; Bawendi, M. G. SlowInjection Growth of Seeded CdSe/CdS Nanorods with Unity Fluorescence Quantum Yield and Complete Shell to Core Energy Transfer. ACS Nano 2016, 10, 3295-3301.

4. $\quad$ Pelton, M.; Ithurria, S.; Schaller, R. D.; Dolzhnikov, D. S.; Talapin, D. V. Carrier Cooling in Colloidal Quantum Wells. Nano Letters 2012, 12, 6158-6163.

5. Guzelturk, B.; Erdem, O.; Olutas, M.; Kelestemur, Y.; Demir, H. V. Stacking in Colloidal Nanoplatelets: Tuning Excitonic Properties. ACS Nano 2014, 8, 12524-12533.

6. Diroll, B. T.; Koschitzky, A.; Murray, C. B. Tunable Optical Anisotropy of Seeded CdSe/CdS Nanorods. J. Phys. Chem. Lett. 2014, 5, 85-91.

7. Diroll, B. T.; Dadosh, T.; Koschitzky, A.; Goldman, Y. E.; Murray, C. B. Interpreting the Energy-Dependent Anisotropy of Colloidal Nanorods Using Ensemble and Single-Particle Spectroscopy. J. Phys. Chem. C 2013, 117, 23928-23937.

8. Vezzoli, S.; Manceau, M.; Leménager, G.; Glorieux, Q.; Giacobino, E.; Carbone, L.; De Vittorio, M.; Bramati, A. Exciton Fine Structure of CdSe/CdS Nanocrystals Determined by Polarization Microscopy at Room Temperature. ACS Nano 2015, 9, 7992-8003. 